level. During the process was verified that although this methodology was systematic and rigorous, was flexible and allowed adapting independently the guideline the nature of the disease, life cycle, and the information's circulation.

Discussion Leading the developing process of guidelines. patients by systematic and flexible methodologies allow to make a better sizing of the process incorporating valid contextual factor, suggests the need to be validated and involve patients in the process.

\section{P236 DOCUMENTATION OF INTELLECTUAL CONFLICTS OF INTERESTS PROVED CRITICAL IN A CLINICAL PRACTICE GUIDELINE}

${ }^{1} \mathrm{E}$ Akl, ${ }^{2} \mathrm{P}$ El-Hachem, ${ }^{3} \mathrm{H}$ Abou-Haidar, ${ }^{4} \mathrm{G}$ Guyatt. ${ }^{1}$ American University of Beirut, Beirut, Lebanon; 'University of Balamand, Beirut, Lebanon; ${ }^{3}$ McGill University, Montreal, Canada; ${ }^{4}$ McMaster University, Hamilton, Ontario

\section{0:1136/bmjqs-2013-002293.220}

Background The American College of Chest Physicians (ACCP) Antithrombotic Guidelines (AT9) addressed both financial and intellectual COI, and restricted panellists from voting on recommendations on which they declared a primary conflict. The extent to which intellectual COI restricted participation beyond financial COI is uncertain.

Objective The objective is to describe financial and intellectual COI among AT9 panellists and assess their overlap.

Methods The AT9 executive committee developed definitions and categorizations of primary and secondary financial and intellectual COI. panellists reported, for each recommendation, their financial and intellectual COIs. We analysed their declarations. Results Among 102 panellists, the average number of recommendations for which panellist declared COI was: 2.1 (SD 5.7) for secondary financial COI, 1.7 (SD 3.5) for primary financial COI, 5.0 (SD 9.9) for secondary intellectual COI, and 2.5 (SD 5.0) for primary intellectual COI. Of the 102 panellists 37 $(36 \%)$ declared a primary intellectual but no primary financial COI for at least one recommendation. Among 431 recommendations, the average number of panellists per recommendation who declared COI was: 0.5 (SD 0.8) for secondary financial COI, 0.4 (SD 0.9) for primary financial COI, 1.2 (SD 1.2) for secondary intellectual COI, and 0.6 (SD 1.2) for primary intellectual COI. In 63 recommendations $(23 \%)$ at least one panellist had a primary intellectual COI but no primary financial COI

Conclusion A substantial number of declarations resulted in restrictions based on intellectual COI in the absence of financial COI.

\section{P239 BREAKING NEW GROUND TO CLOSE THE GAPS BETWEEN PHYSCIAN'S KNOWLEDGE AND PRCATICE}

R Bhushan, D Gist. American Academy of Dermatology, Schaumburg, USA

\section{0:1136/bmjqs-2013-002293.221}

Background Effective dissemination of evidence-based clinical practice guidelines (EBCPG) help clinicians practice evidencebased-medicine, close practice gaps, thus improving medical care.

Objectives To determine the effectiveness of an educational intervention to measure changes in practice/self reported performance improvement for the management and treatment of psoriasis and psoriatic arthritis.

Methods EBCPG were leveraged and repurposed as an interactive guideline translational CME course to expand physicians' knowledge base and improve clinician confidence and effectiveness in treating patients with psoriasis and psoriatic arthritis. Participants were given i) an assessment questionnaire before and after the session to measure knowledge and competence, ii) guidelines application tools useful in the clinic. A follow-up assessment questionnaire was conducted one year later to assess if session and application tools were easy to translate in clinic.

Results Approximately 90\% of the participants felt the session improved their knowledge, confidence and will have an impact on their practice. Over $60 \%$ of case vignettes based questions showed significant improvement compared to the pre assessments $\mathrm{p}<0.05$. A one-year follow up indicated that $63 \%$ of the participants had changed their practice after attending the session.

Discussion The session will optimise usability of EBCPG in physician's daily practice and lead to improvement of patient quality of life and help in closing gaps in practice care.

Implications for Guideline Developers/Users EBCPG translational session with application tools is an effective method for implementing clinical guidelines and helps physicians practice evidence-based-medicine enhancing quality patient care.

\section{P240 THE BENEFITS OF A SYSTEMATIC IMPLEMENTATION STRUCTURE FOR EFFECTIVE IMPLEMENTATION SUPPORT}

T Dunnink. Dutch Centre for Child Health (NCJ), Utrecht, Netherlands

10:1136/bmjqs-2013-002293.222

Background Guidelines do not implement themselves. Structural implementation activities are necessary to improve the usage of guidelines by professionals. At a national level this centre oversees the development, implementation, and evaluation of the Preventive Child Health Care (PCHC) guidelines.

Context In The Netherlands, PCHC is provided by 50 organisations. They use guidelines to improve quality of care, however implementing them, which is their own responsibility, is difficult. They therefore want implementation support, which is then provided by the Dutch Centre for Child Health.

Description of Best Practice To support PCHC organisations implementing guidelines, the Dutch Centre for Child Health developed an implementation structure. The aim of this structure is to provide a systematic implementation through various strategies. The elements of this structure are: a network of implementation coordinators, an implementation toolkit, education and e-learning, a helpdesk, and collaboration with educational institutes and network partners in the youth field. A one year follow-up survey showed that managers and implementation coordinators from PCHC organisations are positive about the support. They want our implementation activities to be continued and even like to see the support extended to guideline maintenance.

Lessons for Guideline Developers, Adaptors, Implementers, and/ or Users organisations want support, to implement guidelines into their own organisation. Beside that they want to know what and when to expect. Our way to achieve that, is to give the support through a clear implementation structure and to provide systematic implementation activities. 